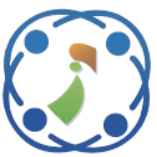

\title{
Cooperative Spectrum Sensing using Rule based Hard Decision and Soft Decision with Bayesian Optimized Support Vector Machine
}

\author{
Anilkumar Dulichand Vishwakarma ${ }^{1 *}$ \\ Girish Ashok Kulkarni ${ }^{1}$ \\ ${ }^{1}$ Kavayitri Bahinabai Chaudhari North Maharashtra University, Jalgaon, India \\ *Corresponding author's Email: anil_karma@yahoo.com
}

\begin{abstract}
Cognitive radio technology has emerged as an operational substitute for increasing the number of users of broadband data services in various frequency bands. This article presents the results of an analysis of the effectiveness of the fusion rule for joint spectrum detection in cooperative spectrum sensing (CSS). The energy detectors-based feature vector is considered for ML training purpose. The paper proposes support vector machine (SVM) based modelling for training and testing in CR. Further Bayesian optimized SVM is proposed to claim higher detection rate. The proposed method yields 0.84 detection rate at 0.1 probability of false alarm.
\end{abstract}

Keywords: Bayesian optimization, CR, ML, SVM.

\section{Introduction}

The evolution of wireless technologies has increased more and more from one generation to the next. From cell phones from the first generation to the $4^{\text {th }}$ generation. The passage from one generation to another required higher speeds and increasingly sophisticated services, which implied a strong demand in terms of the electromagnetic spectrum which is the transmission medium.

The transmission speed criterion being almost solved, several other parameters still posed a problem, such as the protection of data and information related to users, the securing of transmitted messages and the optimization of the use of frequency bands by users because there is a major blockage due to the shortage of spectrum [1].

For this last point, spectrum scarcity, researchers have noticed that a user is not connected 24 hours a day and while they are away, enormous potential in terms of connection time and speed is untapped. It will allow a frequency band to be owned by everyone, not just one user. A so-called primary subscriber can share his channel with another user, called secondary, when he no longer needs it. The primary user can then retrieve his channel at any time, forcing the secondary user to connect elsewhere. The connection to the network is thus optimized and all users are satisfied [2].

Cognitive radio also makes it possible to resolve the problem of the scarcity of spectrum and to guarantee a better quality of service to users. It consists of allowing mobile services to connect opportunistically to frequencies that are underutilized by other users [3].

Thanks to its techniques for learning and decision-making in its knowledge base, it assimilates information about its environment, making it an intelligent, dynamic and adaptive technology to the changes occurring in cognitive radio networks.

Cognitive radio technology appears to be an operational substitute for increasing the number of users in various frequency ranges [4]. The idea targets to address spectrum deficits based on two fundamental characteristics: cognitive ability and ability to reconfigure. Cognitive ability signifies the capacity of a radio to know the environment in which it is functioning, to recognize spectral space that is not fully utilized or at specific intervals, and to communicate its reconfiguration information without disturbing the primary user.

The last decades have seen a considerable 


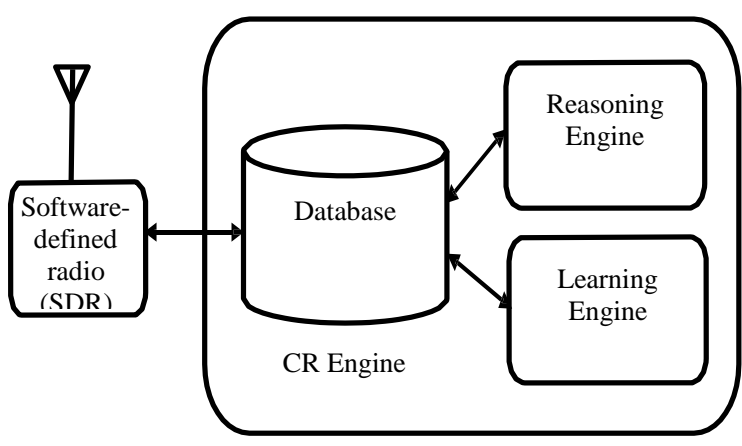

Figure. 1 Machine learning based architecture of cognitive radio

increase in communication techniques. However, the improper use of frequency bands leads to a shortage of spectrum. Cognitive radio appeared to be a solution to this problem [5, 6]. It arranges two types of users:

- Primary User (PU): is the one who holds the spectrum license

- Secondary User (SU): is the user looking for a gap in the spectrum in order to be able to communicate.

The use of cognitive radios provides for spectrum sensing, spectrum occupation information management and dynamic frequency sharing. The execution of these functions requires the implementation of software defined radios with adaptive characteristics, as suggested [7]. Cognitive radio is an intelligent SDR that can, after evaluating the radio frequency environment, record the detected information and associate it with its geographic region and other relevant technical data. The data obtained, duly processed, will allow decisions to be taken regarding spectrum occupation, the start and interruption of irradiation by secondary systems and changes in the configuration of transceivers and operating frequencies so that electromagnetic compatibility in the explored area is guaranteed [8].

In this article, we recommend a powerful machine learning-based spectrum detection algorithm that uses vectors of energy computation for training to distinguish between primary and secondary users. This detection class reliably reflects the action of the PU and the behaviour of the CR user in reaction to it. During the training phase, CR users make decisions at the local level. Local decisions take the form of quantification. The learning phase will be completed when enough data has been collected for the introductory lesson. The SVM algorithm is utilized during the classification phase and the detection class is determined during the testing phase.
Further Bayesian optimized classifier is used to predict the class with higher accuracy.

This paper proposes the machine learning technique using SVM and the average energy received in the current discovery slot for the training and testing as a feature vectors. Further SVM hyperparameters are tuned with Bayesian optimization to perform better classification accuracy.

The purpose of this article is to conduct a comparative exploration of the behaviour of fusion rules for joint detection of spectra using computer simulations. Part II introduces the role of machine learning in cognitive radio networks. Part III contains a system model for the proposed methodology as well as a Bayesian optimized SVM classification. The simulation and its results are described in Section IV. Part V concludes this article.

\section{Machine learning in cognitive radio}

\subsection{Definition}

Machine learning (ML) refers to the design, analysis and application of techniques that allow machines to move through processes and actions, complex or even impractical, learning using more traditional algorithms [9].

- Objective: To automatically extract and use the information present in a dataset.

\subsection{Machine learning based architecture of cognitive radio}

The dynamic spectrum access capabilities of cognitive radios are maximized by the utilization of machine learning as shown in Fig. 1.

- $\quad$ Reasoning Engine: It utilizes the knowledge base to choose the best course of action.

- Learning Engine: It accomplishes knowledge management based on observable information (e.g., channel error rate, channel state information).

- Knowledge Base: maintains system status and available actions. It contains two data structures:

An inference rule is utilized to signify the environment state.

Actions that can be taken to change the state so that the goals of the framework can be reached. 


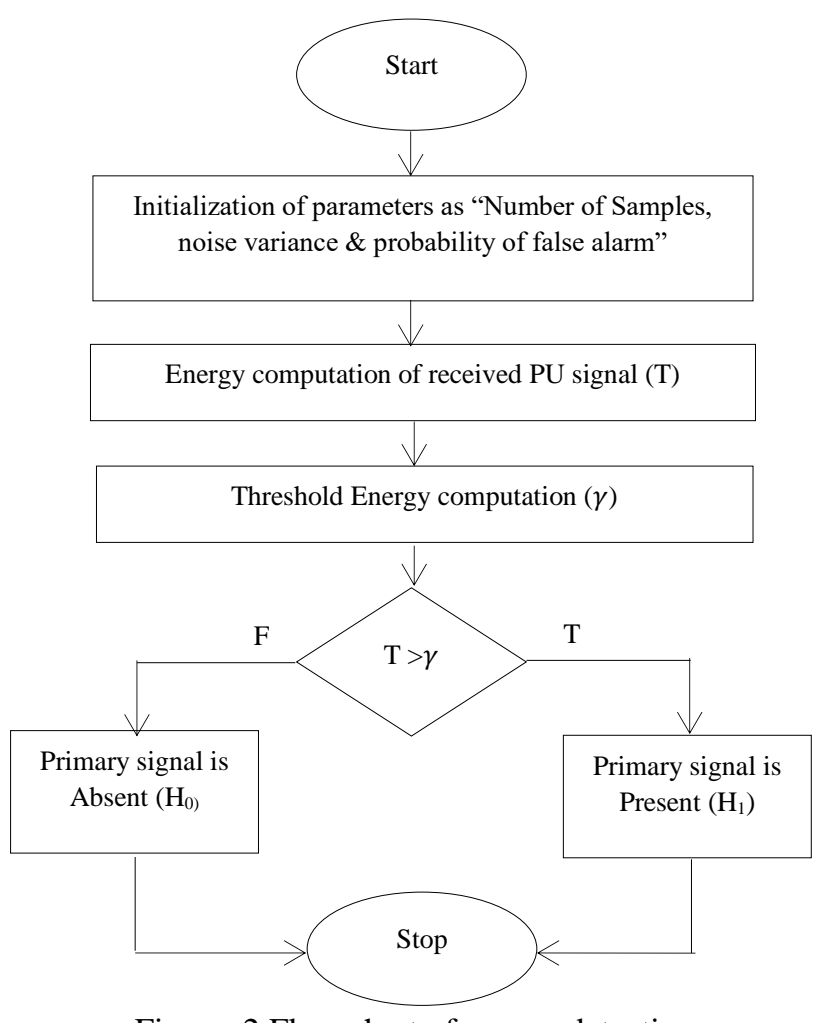

Figure. 2 Flow chart of energy detection

\section{Proposed methodology}

\subsection{System model with energy detection}

The first step for better communication through cognitive radio is the detection of free frequency bands. The presence of a signal will subsequently be characterized by $H_{1}$ and the absence will be by $H_{0}$.

$$
\begin{aligned}
& H_{0}: y(t)=b(t) \\
& H_{1}: y(t)=x(t)+b(t)
\end{aligned}
$$

Where, $y(t)$ is the received signal $x(t)$ : Primary signal and $b(t)$ : Noise

The statistical test of method will then be calculated compared to a threshold $\xi$ predefined for each type in order to determine whether there is the absence or presence of a signal. If: $T \geq \xi$ then the primary signal is present, otherwise it is absent. Energy measurement, also known as a "radiometer", is a blind and incoherent measurement method [10] irrespective of the previous information of the signal.

Its statistical test will be as follows:

$$
T_{E D}=\frac{1}{T} \int_{o}^{T} y(t)^{2} d t
$$

Where: $y(t)$ is the received signal, the probabilities of false alarm and detection in a Gaussian channel are as follows:

$$
\begin{gathered}
P_{d, E D}=Q_{N}(\sqrt{2 T B \rho}, \sqrt{\xi}) \\
P_{f a, E D}=\Gamma\left(T B, \frac{\xi}{2}\right)
\end{gathered}
$$

Where: $\mathrm{T}$ is the period and $\Gamma($.$) is the Gamma$ function, $B$ is the bandwidth and $\rho$ is the signal to noise ratio

If the noise level is greater than that of the signal then its performance is degraded, and is not optimal for noisy signals [11].

\subsection{Cooperative spectrum sensing model}

There is always a need of a control channel among the centralized exchange, adjacent secondary users and CR for both the centralized and distributed techniques. The bandwidth of this control channel is capable of limiting the amount of transmitted data. If all users report their decisions to the synthesis centre or relay them to neighbours, effective detection can lead to congestion of the control channel.

Moreover, two cases are found on the basis of the data pass through the control channel. First, the $u_{k}$ decision bits sent by the secondary user are stored in the fusion centre. The second case is a situation where a CR sends its decision to the appropriate focal point or CR for channel status.

Distributed and centralized schemes use two different rules for the primary user state and for the channels. Each of these is discussed in the next sections. For this, a scenario was deliberated with a secondary cognitive user $\mathrm{K}$, recognizing the presence of the primary user. $\mathrm{N}$ samples of the received signal are independently collected by each of those users.

\subsubsection{Hard decision}

In a complex decision-making structure, a specific CR decides on the presence of a primary user. Bit 1 specifies that the primary user is utilizing the channel spectrum and therefore the CR cannot use it. The spectrum is available at 0 bit and radio can be used it.

- Majority Rule: It is the most famous rule where you have several $\mathrm{N}$ users sending decisions about detecting the status of the local link to the center of the union via the information bits $\mathrm{u} \_\mathrm{k}$, and then decisions about linear combinations of different 
decisions are made by each secondary user, the decision rules are presented in Eq. (6).

$$
D=\sum_{k=1}^{K} u_{k}
$$

If $\mathrm{D} \geq \mathrm{M}$, the fusion center selects $\mathrm{H}_{1}$ which shows that the transmitter channel is enable, elseD $<$ $M$ chooses $\mathrm{H}_{0}$.

The majority rule is an exceptional instance of vote counting rule, where $\mathrm{M}=\frac{\mathrm{K}}{2}$.

Each secondary user has a false alarm and detection probability, which are shown for each user in Eqs. (7) and (8), respectively.

$$
\begin{gathered}
Q_{F A}=P(D \geq M)=\prod_{i=1}^{N}\left(P_{F A, i}\right)^{i}\left(1-P_{F A, i}\right)^{N-i} \\
Q_{D}=P(D \geq M)=\prod_{i=1}^{N}\left(P_{D, i}\right)^{i}\left(1-P_{D, i}\right)^{N-i}
\end{gathered}
$$

Two new rules can be derived from the combination rules that are presented below.

- AND Rule: The decision bits of each secondary user is the fundamental basis of this rule. These bits are associated with the channel status. The fusion center selects hypothesis $\mathrm{H} \_1$ if all users $\mathrm{k}=\mathrm{N}$ detect the signal, i.e. decision bit of the primary user is $\mathrm{u} \_\mathrm{k}=1$ in the frequency band of interest selected for all users, otherwise if the decision bit is with the primary user equals $\mathrm{u} \_\mathrm{k}=0$, and the chosen hypothesis is H_0. The probabilities were measured by utilization of Eqs. (9) and (10) for the probability of detection and the probability of a false alarm, respectively.

$$
\begin{gathered}
Q_{D}=\prod_{n=1}^{N} P_{D, n} \\
Q_{F A}=\prod_{n=1}^{N} P_{F A, n}
\end{gathered}
$$

If the probabilities are the same for all users, then the probability of detection and the probability of a false alarm can be determined as indicated in Eqs. (11) and (12).

$$
Q_{D}=\left(P_{D}\right)^{N}
$$

$$
Q_{F A}=\left(P_{F A}\right)^{N}
$$

- $\quad$ OR Rule: This rule is effective if at least one subscriber station identifies the occurrence of a PU signal in the desired band. The probabilities of false alarms and false alarms were determined by Eqs. (13) and (14), respectively.

$$
\begin{aligned}
& Q_{F A}=1-\prod_{n=1}^{N}\left(1-P_{F A, n}\right) \\
& Q_{D}=1-\prod_{n=1}^{N}\left(1-P_{D, n}\right)
\end{aligned}
$$

If the user probabilities are equal, we have:

$$
\begin{aligned}
& Q_{F A}=1-\left(1-P_{F A}\right)^{N} \\
& Q_{D}=1-\left(1-P_{D}\right)^{N}
\end{aligned}
$$

\subsubsection{Soft decision}

The secondary user submits the results of the statistical test $\mathrm{u}_{\mathrm{k}}$ to the $\mathrm{FC}$ deprived of executing any previous analysis or procedure. The information established at the FC is handled and equated with the limit values set by the secondary user, which allows decisions on the absence or presence of activity of the primary user.

With regard to the procedures accomplished on the information in the smelter, the exists two types of soft decision techniques discussed below.

- Selection Combining (SC): In this method, highest SNR branch is selected by the fusion centre. As shown in Eq. (17), where $\gamma_{-} \mathrm{k}$ is the SNR for each leg and $\gamma_{-} \mathrm{SC}$ is the selected SNR.

$$
\gamma_{S C}=\max \left(\gamma_{1}, \gamma_{2}, \ldots, \gamma_{K}\right)
$$

For an AWGN channel, the probabilities of false alarms and detection are deliberated in Eqs. (18) and (19), respectively.

$$
\begin{gathered}
Q_{F A, S C}=\frac{\Gamma\left(m, \frac{\lambda}{2}\right)}{\Gamma(m)} \\
Q_{D, S C}=Q_{m}\left(\sqrt{2 \gamma_{S C}}, \sqrt{\lambda}\right)
\end{gathered}
$$

Where $Q_{m}(.,$.$) is the general Marcum function,$ $\Gamma($.$) and \Gamma(.,$.$) are the comprehensive and$ inadequate Gamma function, $\lambda$ is user-defined 
threshold value. $\mathrm{m}$ is the product of time and bandwidth, $\mathrm{m}=\mathrm{TW}$.

- $\quad$ Maximum Ratio Combining (MRC): In such type of rule, each estimated power or signal energy received by each secondary user is weighted byw_k $=\gamma_{-} \mathrm{k} /\left(1+\gamma_{-} \mathrm{k}\right)$ where $\gamma_{-}$kis the SNR of each user. Consequently, cognitive radios with higher SNR receive more weight. At the fusion center, an arrangement of weights and user energies was accomplished to achieve a statistical test used with respect to the detection thresholds presented for this combination in Eq. (20) and in reference [12].

$$
E_{M R C}=\sum_{k=1}^{K} w_{k} E_{k}
$$

The detection probability and probability of false positives for MRC are shown in Eqs. (21) and (22).

$$
\begin{gathered}
Q_{D, M R C}=Q_{m}\left(\sqrt{2 \gamma_{M R C}}, \sqrt{\lambda}\right) \\
Q_{F A, M R C}=\frac{\Gamma\left(m K, \frac{\lambda}{2}\right)}{\Gamma(m)}
\end{gathered}
$$

The signal-to-noise ratio of this technique is given by Eq. (23).

$$
\gamma_{M R C}=\sum_{k=1}^{K} \gamma_{k}
$$

\subsection{Hayesian optimized support vector machine based proposed machine learning model for css}

This spectrum detection system aims to increase the capability of detection for PUs in various environments to improve the detection of spectral holes. The initial objective provides security of primary user's data from destructive interference and is a key constraint defined by IEEE 802.21, a standard for accessing white space in TV. The second goal is to leverage the frequency access capabilities and permit $\mathrm{CR}$ users to transmit data.

For the $\mathrm{i}^{\text {th }}$ user $\mathrm{CR}$ in the $\mathrm{w}^{\text {th }}$ detection interval, channel availability is determined based on the energy vector $R_{i}{ }^{w}$. To properly coordinate PU activities, it is necessary to study the PU behaviour. Therefore, the energy vector in our case is similar to the resource vector in the machine learning environment.
To build a classifier, i.e. to divide the existing acquisition rate into occupied channel $\left(\mathrm{H}_{1}\right)$ or available channel $\left(\mathrm{H}_{0}\right)$ classes, a learning step is required. Each $\mathrm{CR}$ user provisions an energy vector of $\mathrm{W}$ dimension, where $\mathrm{W}$ is the duration of the training phase. The segment arrangement is utilized during the learning phase; one slot has a transmission phase and a detection phase. During the training phase, there is a slot W. During the classification phase, these vectors are placed in the classifier, where the existing acquisition rate is matched with the earlier deposited acquisition rate to choose between $\mathrm{H}_{0}$ and $\mathrm{H}_{1}$.

In this work, the $\mathrm{CR}$ user first learns the behaviour of the PU by comparing the resulting quantized energy vector, called the detection rate, with the correct state of the PU.

The fusion centre determines the consistent arrangement of local user decisions and ACK, which determines the actual prominence of the PU. There are diversified roles of CR users in the classification phase and the training phase. During the training phase, detection reports are allocated to detection classes based on the equivalent $\mathrm{CR}$ user behaviour and authentic PU action. During the classification step, the detection report is categorized into one of the detection classes utilizing the SVM. For accurate calculations, an additional optimal Bayesian classifier is used.

\subsubsection{Training phase}

At this stage, the functional environment is captured by measuring the behaviour of CR users in relation to the development of PU activities. The $\mathrm{i}^{\text {th }}$ user CR generates discovery reports, makes local decisions based on the average energy received in the current discovery slot, sends local decisions to the FC, and based on the results of the FC and ACK status, reports from discovery to discovery. This section details these steps.

Alternatively, the energy received in the $\mathrm{w}^{\text {th }}$ detection interval in the $\mathrm{i}^{\text {th }}$ user's $\mathrm{CR}$ is represented as follows:

$$
Y_{i, w}=\frac{\sum_{k=1}^{n} X_{k}, w, i}{n}
$$

\subsubsection{Classification phase}

The optimization of discrete and continuous variables provides the enhancement in rating and flexibility with problems to deal with several types of data. The SVM is a statistical system based classification method. Though, it has some negative 
Table 1. Simulation parameters

\begin{tabular}{|c|c|}
\hline Parameters & Value \\
\hline Bandwidth & $\mathrm{w}=5 \mathrm{MHz}$ \\
\hline Sampling Frequency & $\mathrm{f}_{\mathrm{s}}=10 \mathrm{MHz}$ \\
\hline $\begin{array}{c}\text { Noise Power Spectral } \\
\text { Density }\end{array}$ & $\begin{array}{c}\eta_{0}= \\
-152 \mathrm{dBm} / \mathrm{Hz}\end{array}$ \\
\hline PU active probability & $\mathrm{p}_{(\mathrm{H} 1)}=0 .=0.5$ \\
\hline PU transmission power & $0.1 \mathrm{~mW}$ \\
\hline SU1 $\rightarrow$ PU distance & $500 \mathrm{~m}$ \\
\hline SU2 $\rightarrow$ PU distance & $750 \mathrm{~m}$ \\
\hline SU3 $\rightarrow$ PU distance & $1000 \mathrm{~m}$ \\
\hline
\end{tabular}

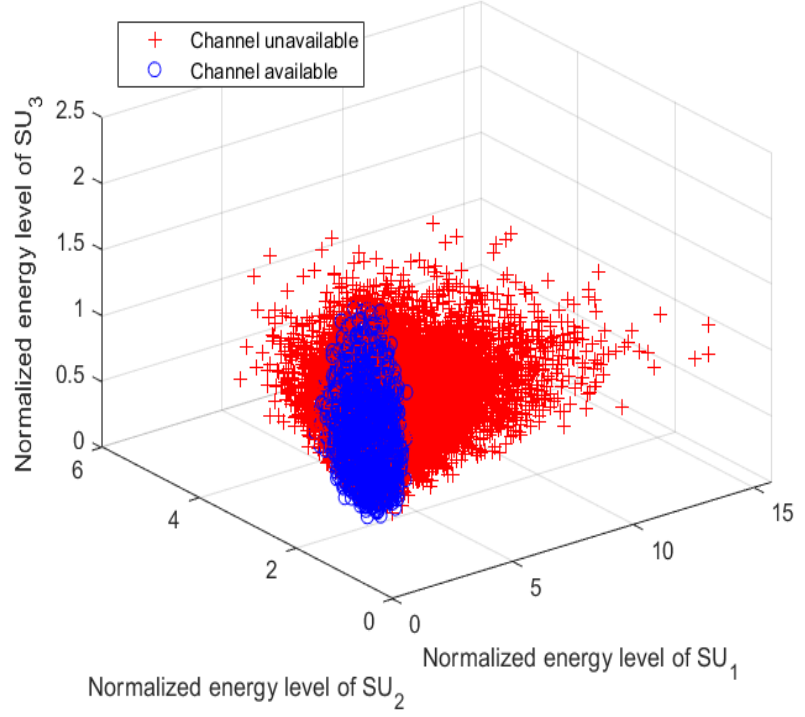

Figure 3. Received energy level

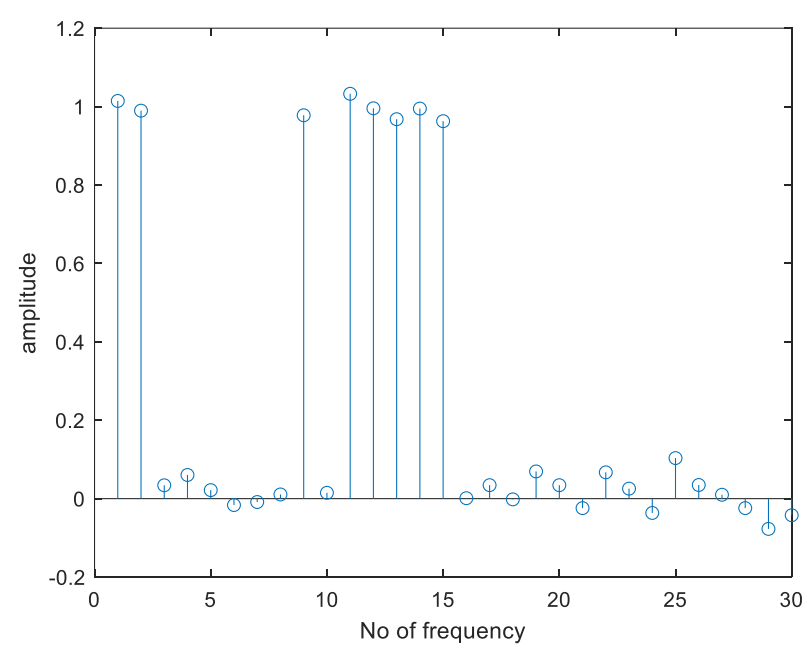

Figure. 4 Spectrum sensing subbands at 30 SNR under AWGN channel

aspects of choosing and configuring inputs. The parameters of SVM are optimized by detecting the deviations in constant value. This process causes the information removal that decreased the classification accuracy.

The proposed algorithm is related to twoparameter SVM optimization. Parameters: i) weight,
$\mathrm{C}$; and ii) fundamental functions. Weights are a tradeoff between misclassifying some elements and classifying others correctly when the kernel is utilized to add SVM parameters and choose input properties. Here, the SVM parameters are optimized by Bayesian Optimization [13].

\subsubsection{Bayesian optimized-SVM algorithm}

Input: initialize input for $C, \gamma, k$ solution

Output: best value for $C$ and $\gamma$ for higher classification accuracy

Begin

For kloop

Execute SVM model for $k$ solutions

$T=\operatorname{Sort}(S 1, \ldots, S k)$

while classification accuracy $\neq 100 \%$ do

forj $=1$ to nloop

find $C$ and

call SVM model to evaluate newly generated solutions

end

$T=$ Best $($ Sort $S 1, \ldots S k+m), k)$

end

End

Where, $k$ is the size of the solution file, $m$ is the number of models utilized to produce the solution, $q$ is the algorithm parameter for controlling the variation of the search procedure, $C$ is the smoothing or uniform stock parameter, $\gamma$ is the width parameter also known as the margin or kernel function parameter.

\section{Simulation results}

The above figure shows the corresponding channel status and energy level at receiver on three subbands.

Fig. 5 shows the ROC curve graph of energy detection technique with $\mathrm{P}_{\mathrm{d}}$ vs. $\mathrm{P}_{\mathrm{fa}}$ at various SNR values $(\mathrm{SNR}=-5 \mathrm{~dB}, \mathrm{SNR}=-10 \mathrm{~dB}, \mathrm{SNR}=-15 \mathrm{~dB} \&$ $\mathrm{SNR}=-20 \mathrm{~dB}$ ), For Noise variance $=0.2$, No. of Samples $=500$.

In Fig. 5, the probability of detection $\mathrm{P}_{\mathrm{d}}$ is plotted under $H_{1}$ against the probability of false alarm $\mathrm{P}_{\mathrm{fa}}$ under $H_{0}$ where $\mathrm{P}_{\mathrm{fa}}$ changes from 0.01 to the desired value of 0.1 . The values $\mathrm{P}_{\mathrm{d}}$ for various values of $\mathrm{P}_{\mathrm{fa}}$ over varying SNR levels are represented in Table 2. From Table 2, it is clear that the probability of detection at the $-5 \mathrm{~dB}$ SNR level is nearly 0.185 to 0.393 greater than the $P_{d}$ at $-10 \mathrm{~dB}$ SNR level at different points. As the values of $P_{d}$ at SNR level, $-15 \mathrm{~dB}$ and $-20 \mathrm{~dB}$ are approximately the same but approximately 0.193 to 0.420 smaller than SNR level is -5 . 


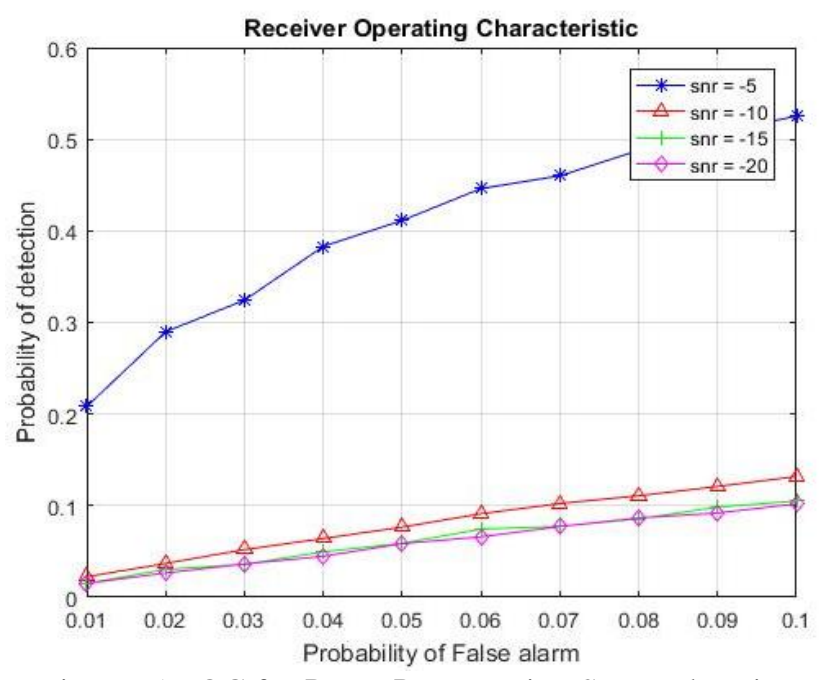

Figure. 5 ROC for $P_{d}$ vs. $P_{f a}$ at varying $S N R$ values in energy detection technique

Table 2. Analysis of ROC for $\mathrm{P}_{\mathrm{d}}$ vs. $\mathrm{P}_{\mathrm{fa}}$ at varying $\mathrm{SNR}$ values in energy detection technique

\begin{tabular}{|c|c|c|c|c|}
\hline \multirow{2}{*}{$\begin{array}{c}\text { Probability } \\
\text { of false } \\
\text { alarm }\left(\boldsymbol{P}_{\boldsymbol{f} \boldsymbol{a}}\right)\end{array}$} & $\begin{array}{c}\text { PNR }= \\
\mathbf{- 5}\end{array}$ & $\begin{array}{c}\text { SNR }= \\
\mathbf{- 1 0}\end{array}$ & $\begin{array}{c}\text { SNR }= \\
\mathbf{- 1 5}\end{array}$ & $\begin{array}{c}\text { SNR }= \\
\mathbf{- 2 0}\end{array}$ \\
\hline 0.0100 & 0.2082 & 0.0224 & 0.0152 & 0.0152 \\
\hline 0.0200 & 0.2898 & 0.0370 & 0.0304 & 0.0266 \\
\hline 0.0300 & 0.3244 & 0.0520 & 0.0360 & 0.0364 \\
\hline 0.0400 & 0.3830 & 0.0642 & 0.0496 & 0.0448 \\
\hline 0.0500 & 0.4114 & 0.0766 & 0.0590 & 0.0586 \\
\hline 0.0600 & 0.4462 & 0.0912 & 0.0744 & 0.0660 \\
\hline 0.0700 & 0.4602 & 0.1024 & 0.0774 & 0.0772 \\
\hline 0.0800 & 0.4878 & 0.1108 & 0.0856 & 0.0866 \\
\hline 0.0900 & 0.5070 & 0.1210 & 0.0984 & 0.0920 \\
\hline 0.1000 & 0.5254 & 0.1318 & 0.1052 & 0.1020 \\
\hline
\end{tabular}

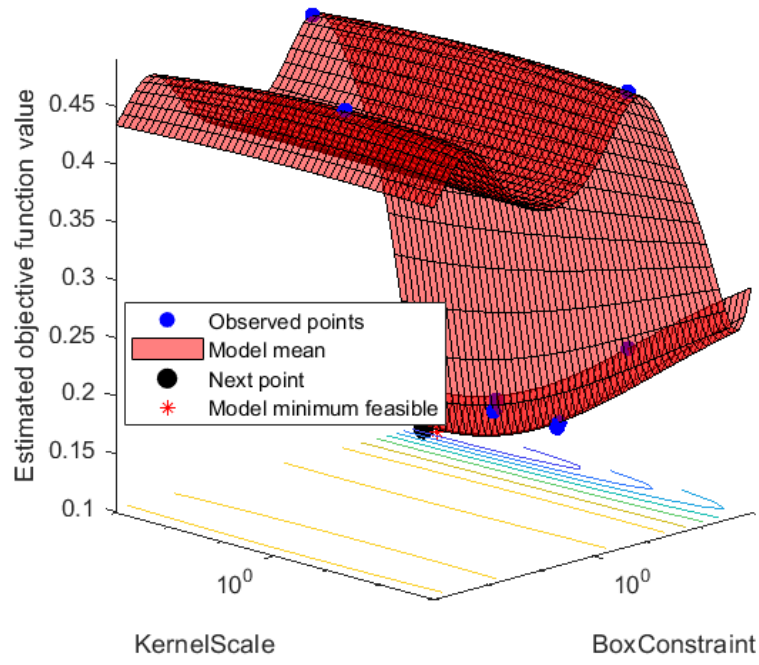

Figure. 6 Objective function model

So from the ROC curve in Fig. 5, it is clear that the probability of detection is higher at the $-5 \mathrm{~dB}$ SNR level when compared with various SNR levels

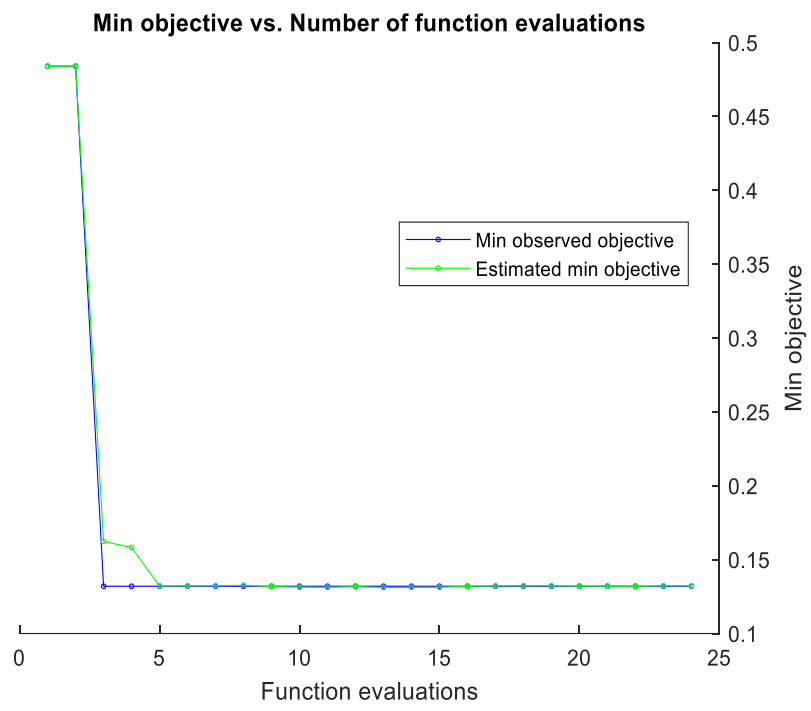

Figure. 7 Min. objective vs. number of function evaluations

Table 3. Simulation parameters for machine learning

\begin{tabular}{|c|c|}
\hline Parameters & Value \\
\hline $\mathrm{M}$ & $10^{4}$ Samples. \\
\hline $\mathrm{M}$ & $2 ;$ Number of Primary users (PU) \\
\hline $\mathrm{N}$ & $2 ;$ Number of Secondary users (SU) \\
\hline Omega & $30 ;$ Number of samples \\
\hline Sigma & $2 ;$ Noise power \\
\hline Iter & $1000 ;$ Learning cycle \\
\hline $\mathrm{R}$ & $1000 ;$ Number of iteration \\
\hline Training Size & $100 ;$ Size of training set \\
\hline Beta & $2 ;$ Threshold of detection \\
\hline$K_{S}$ & $\begin{array}{c}2 ; \text { Number of subbands (Assume } \\
\text { every SU takes one subband when } \\
\text { channel is available) }\end{array}$ \\
\hline
\end{tabular}

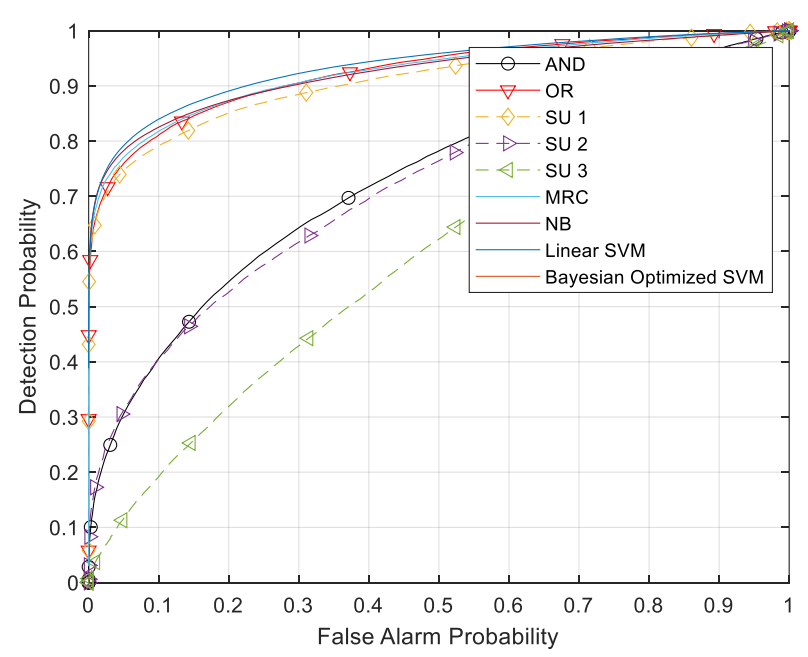

Figure. 8 ROC curves for the different techniques training with 100 training sample

(Example; $-10 \mathrm{~dB},-15 \mathrm{~dB}$, and $-20 \mathrm{~dB}$ ) which verifies the better performance at higher SNR using energy 
Table 4. Comparison of proposed with existing method

\begin{tabular}{|c|c|c|}
\hline Methods & $\begin{array}{c}\text { Probability } \\
\text { of Detection } \\
\left(\boldsymbol{P}_{\boldsymbol{d}}\right)\end{array}$ & $\begin{array}{c}\text { Probability } \\
\text { of False } \\
\text { Alarm }\left(\boldsymbol{P}_{\boldsymbol{f} \boldsymbol{a}}\right)\end{array}$ \\
\hline $\begin{array}{c}\text { Proposed } \\
\text { Bayesian- } \\
\text { optimized SVM }\end{array}$ & 0.84 & 0.1 \\
\hline Hossain et al. [13] & 0.7 & 0.1 \\
\hline Ma et al. [14] & 0.5 & 0.1 \\
\hline Tavares et al. [15] & 0.8 & 0.1 \\
\hline
\end{tabular}

detection technique.

To estimate the performance of the previous model in deriving the channel state, we performed a Monte Carlo simulation with a $5 \times 104$ implementation, with the scenario shown in Fig. 8, with PU and $3 \mathrm{SU}$ and parameters in Table 1 at additive Rayleigh fading channel. We compared the optimized SVM Bayesian method with traditional AND, OR, and MRC analysis methods. For optimized Bayesian SVM, we consider linear and Gaussian kernel functions.

Through graphical examination, we can see the superior performance limit determined by the MRC method, followed by a Bayesian optimized SVM with a linear kernel. In addition to the cooperative method, we have a graph of the ROC curve obtained by determining the individual energies in each SU. Due to the difference in distance from the PU, the resulting average signal-to-noise ratios are $2 \mathrm{~dB}, 9 \mathrm{~dB}$ and $14 \mathrm{~dB}$. This difference can be seen from the channel recognition characteristics shown by each SU.

The proposed work explores the potential of cooperative spectrum sensing (CSS) based on machine learning in classification speed, training time, and classification performance. This is further improved by Bayesian optimized SVM.

Authors of [11] mainly focuses on, twodimensional distance vector which is transformed by an m-dimensional energy vector permitting to the distance extent between vectors. Furthermore, KNN is applied to perform the classification. The authors of [11] majorly focus on probability vector and distance vector as feature vectors, but in the case of poor signal and less users the proposed features don't perform well as compare to energy detector-based method. Authors of [14] proposed Cognitive radio performance Fuzzy logic and naïve Bayes classifier. The above method is implemented in VANET platform and achieve detection is 0.5 at probability of false alarm is set at 0.1. Authors of [15] considered centralized cooperative spectrum sensing (CCSS) methods for cognitive radio networks using energy detector method. The paper proposes SVM based modelling and achieve 0.80 probability of detection, whereas proposed method yields 0.84 detection rate at same reference.

\section{Conclusion}

Cognitive radio technology appears to be an operational substitute for increasing the number of users in various frequency bands. The idea aims to address spectrum deficits based on two fundamental characteristics: cognitive ability and ability to reconfigure. Cognitive ability refers to the capacity of a radio to know the environment in which it is operating, to recognize spectral space that is not fully utilized or at specific intervals, and to communicate its reconfiguration information

Without disturbing the primary user utilized spectrum. Detectors HARD types (with rules AND, OR) and SOFT types (SVM and Bayesian optimizedSVM) were evaluated in scenarios in which SU had different SNR values, that are a direct consequence of the SU position regarding the PU, whose position is unknown most of the time. As a future work, we can execute deep learning network for cooperative cognitive radio spectrum and non-cooperative methods for cooperative cognitive radio spectrum.

\section{Conflicts of interest}

The authors declare no conflict of interest.

\section{Author contributions}

The paper Conceptualization, methodology, software, validation, formal analysis, investigation, resources, data curation, writing-original draft preparation, writing-review and editing, visualization have been done by first author. The supervision and project administration have been done by second author.

\section{References}

[1] A. A. Khan, M. H. Rehmani, and A. Rachedi, "Cognitive-radio-based internet of things: Applications, architectures, spectrum related functionalities, and future research directions", IEEE Wireless Communications, Vol. 24, No. 3, pp. 17-25, 2017.

[2] F. Hu, B. Chen, and K. Zhu, "Full spectrum sharing in cognitive radio networks toward $5 \mathrm{G}$ : A survey", IEEE Access, Vol. 6, pp. 1575415776, 2018.

[3] D. Tarek, A. Benslimane, M. Darwish, and A. M. Kotb, "Survey on spectrum sharing/allocation 
for cognitive radio networks Internet of Things", Egyptian Informatics Journal, 2020.

[4] A. A. Astaneh, and S. Gheisari, "Review and comparison of routing metrics in cognitive radio networks", Emerging Science Journal, Vol. 2, No. 4, pp. 191-201, 2018.

[5] H. Khaled, I. Ahmad, D. Habibi, and Q. V. Phung, "A Secure and Energy-Aware Approach For Cognitive Radio Communications", IEEE Open Journal of the Communications Society, Vol. 1, pp. 900-915, 2020.

[6] R. Struzak, "Cognitive radio, spectrum, and evolutionary heuristics", IEEE Communications Magazine, Vol. 56, No. 6, pp. 166-171, 2018.

[7] M. M. Kaidenko and D. V. Roskoshnyi, "Software defined radio in communications", In: Proc. of International Conf. On Information and Telecommunication Technologies and Radio Electronics, Springer, Cham, pp. 227-238, 2018.

[8] R. Akeela and B. Dezfouli, "Software-defined Radios: Architecture, state-of-the-art, and challenges", Computer Communications, Vol. 128, pp. 106-125, 2018.

[9] D. Tarek, A. Benslimane, M. Darwish, and A. M. Kotb, "Cognitive radio networks channel state estimation using machine learning techniques", In: Proc. of International Conf. On $15^{\text {th }}$ International Wireless Communications \& Mobile Computing Conference (IWCMC), IEEE, pp. 342-347, 2019.

[10] K. Clark, M. T. Luong, C. D. Manning, and Q. $\mathrm{V}$. Le, "Semi-supervised sequence modeling with cross-view training", arXiv preprint arXiv:1809.08370, 2018.

[11] S. U. Jan, V. H. Vu, and I. Koo, "Throughput maximization using an SVM for multi-class hypothesis-based spectrum sensing in cognitive radio", Applied Sciences, Vol. 8, No. 3, p. 421, 2018.

[12] A. Gupta and S. Majumder, "Cognitive radio spectrum classification using FLA-SVM", In: Proc. of International Conf. On $9^{\text {th }}$ Annual Information Technology, Electromechanical Engineering and Microelectronics Conference (IEMECON), IEEE, pp. 144-149, 2019.

[13] M. A. Hossain, R. M. Noor, K. L. A. Yau, S. R. Azzuhri, M. R. Z'aba, I. Ahmedy, and M. R. Jabbarpour, "Machine Learning-Based Cooperative Spectrum Sensing in Dynamic Segmentation Enabled Cognitive Radio Vehicular Network", Energies, Vol. 14, No. 4, p. 1169, 2021.

[14] S. Ma and J. Shi, "Performance improvement for machine learning-based cooperative spectrum sensing by feature vector selection", IET Communications, Vol. 14, No. 7, pp. 1081-1089, 2019.

[15] C. H. A. Tavares and T. Abrão, "Bayesian estimators for cooperative spectrum sensing in cognitive radio networks", In: Proc. of International Conf. of URUCON, IEEE, pp. 1-4, 2017. 